Revista Iberoamericana, Vol. LXX, Núms. 208-209, Julio-Diciembre 2004, 773-793

LA CAMPANA DE PALO (1926-1927):
UNA ACCIÓN EN TRES TIEMPOS

\author{
POR \\ Patricia M. Artundo \\ Instituto de teoría e historia del arte "Julio E. Payro" \\ Universidad de Buenos Aires
}

El anarquismo argentino es una bolsa de gatos, cuya boca es apretada por la mano de la burguesía: se muerden entre ellos, se tarasconean, se pelean y nadie atina a morder la mano del burgués que los esclaviza. ${ }^{1}$

Con estas palabras Atalaya (Alfredo Chiabra Acosta, 1889-1932) resumía su visión del anarquismo argentino. En ellas se puede descubrir la profunda decepción que sentía quien había sido partícipe activo del movimiento anarquista desde principios de los años veinte, en particular, desde su trabajo como editor del Suplemento Semanal (1922-1926) de La Protesta.

El cierre para esa relación tiene fecha precisa: el 15 de octubre de 1926 cuando fue dejado cesante; corte abrupto que había sido adelantado meses antes cuando su labor quedó reducida a su mínima expresión. ${ }^{2}$ Entre sus camaradas anarquistas finalmente había vencido la idea de que, desde un punto de vista práctico, el arte carecía de valor revolucionario: ellos desconfiaban de la importancia que él podía revestir para el proletariado urbano en función de su adoctrinamiento.

\footnotetext{
${ }^{1}$ [Atalaya]. "El anarquismo argentino". Texto sin datar, posterior a 1926. Archivo Atalaya. Propiedad Albino Fernández. [Reflexiones, 36]. Como parte de la beca que me fuera otorgada por el Fondo Nacional de las Artes (FNA) tuve a mi cargo la organización de este Archivo. Véase: Artundo, Atalaya.... Compuesto por más de 1.000 documentos, estos fueron organizados a partir de los diversos temas y géneros abordados por Atalaya, asignándoles una numeración corrida. En adelante los textos pertenecientes a este Archivo serán citados como Archivo Atalaya (AA), indicando a continuación el tipo de documento y su número de orden, como en este caso: "AA. Reflexiones, 36”

${ }^{2}$ Hasta entonces había comprendido crítica y notas de arte, sueltos, traducciones, cuentos, bibliografía, textos doctrinarios y de divulgación, selección de obras a reproducir. Su correspondencia con Luis Falcini informa acerca de las alternativas por las que pasó su relación con La Protesta. Véanse: Cartas datadas “5 noviembre/26” (MNBA/26) y “4 agosto de 1926” (MNBA/39). Epistolario Atalaya-Falcini. Donación Falcini. Archivo Documental. Museo Nacional de Bellas Artes (MNBA). La edición de estas cartas se encuentra agregada al informe de Artundo, 2001. En adelante estas cartas serán citadas por su localización actual (MNBA) y el número con el que fueron ingresadas como parte de la Donación Falcini.
} 
Esa desconfianza se había manifestado desde el momento mismo de su incorporación al Suplemento; en 1922, Atalaya había tenido que defenderse de ciertas críticas y en “Labor cultural y orientación artística” había afirmado que:

[...] El propósito de lucha no debe excluir al de una siembra cultural para el futuro. Todo lo que hagamos para educar al sentimiento y el gusto de los hombres, será labor beneficiosísima.

[...] Entre muchos anarquistas se teme que esto signifique una desviación de la línea recta que se le ha trazado a la propaganda revolucionaria; y que, a veces, la publicación de una biografía de un hombre como Tolstoy o Leonardo Da Vinci, es salirse de los cánones subversivos preestablecidos. Sin embargo, en cuestiones culturales, nadie debería ser más sectario que nosotros.

Y hay que comprender que la base de la futura cultura proletaria, sólo se podrá formar con las obras de los verdaderamente grandes, quienes por su genialidad, fueron sencillos y, por lo mismo, resultan los más accesibles al pueblo. (2)

En la base de los cuestionamientos que tenían lugar en el seno del diario se encontraba una de las particularidades del anarquismo: allí todo estaba sujeto a discusión y esto explica también los constantes cambios producidos en el cuerpo de su redacción. ${ }^{3}$ Para Eduardo G. Gilimón, los redactores:

[...] de una publicación anarquista están colocados en una situación poco grata, por cuanto que los lectores son apasionados, toman una ingerencia en el diario que resulta molesta y dados los matices tan varios que entre los anarquistas existen, siempre hay un número considerable de descontentos con la redacción.

Otras publicaciones no tienen nada que temer del público lector. El que lee un diario, si en él encuentra algo que no le gusta, lo pasa por alto y sigue leyendo lo demás.

En el campo anarquista las cosas pasan de otro modo.

Lo que no agrada se comenta, se critica y llega hasta promover actos de desagrado (5253).

Si en esta breve introducción hemos evitado abordar directamente nuestro tema, ello se debe a que La Campana de Palo. Periódico mensual. Bellas Artes y Polémica hizo su reaparición precisamente en el momento de ruptura que hemos señalado; por tal motivo resulta difícil llegar a entender esta nueva etapa ignorando la relación de Atalaya con el anarquismo. Lo mismo puede afirmarse respecto de Carlos Giambiagi (1887-1965), su compañero al frente de la revista; él también compartía su oposición a los mecanismos de funcionamiento de la sociedad: sus frecuentes escapes a San Ignacio y su vida en la selva

${ }^{3}$ Para una historia del diario, véase: Abad de Santillán, 1927 y Quesada, 1974. Recientemente el CeDInCI publicó en versión digital el Certamen Internacional de La Protesta (2002). Luego de finalizado este trabajo para Revista Iberoamericana, fue publicado el libro de Juan Suriano, Anarquistas: cultura y política libertaria en Buenos Aires. 1890-1910. Buenos Aires: Manantial, 2001, que resulta de imprescindible consulta. Varios de los puntos que tratamos aquí son desarrollados con detenimiento por Suriano y lamento no haberlo conocido antes dado que además su lectura resulta sugerente en muchos sentidos. 
misionera llevaban implícita su abierta crítica y rechazo a ellos. En una carta a su amigo Atalaya, fechada el 22 de septiembre de 1921, este pintor y grabador resumía la visión que tenía de sí mismo: “[...] somos anarquistas, en ese sentido de críticos agudos de esperanza y fe en el porvenir. Como críticos escépticos en toda esa serie de iniciativas que atañen a cuestiones transitorias; y místicos por la fuerza de nuestra fe en lo que ha de venir” (212).

En el caso de Atalaya, él puede ser considerado como uno de aquellos intelectuales críticos que segregados de la sociedad encuentran en algún momento un espacio en o cerca de los partidos y movimientos políticos. Para Michael Walzer, estos críticos sociales "son apenas extraños en su nuevo ambiente. Los partidos y los movimientos son creaciones de personas muy semejantes a ellos mismos. En la medida en que siguen siendo críticos, críticos ahora de sus nuevos camaradas, no están tanto alienados como dificultosamente integrados [...]” (16) [énfasis agregado].

El incorporar esta nueva perspectiva de estudio en nuestra lectura de La Campana de Palo, resulta imprescindible no sólo para comprender la propuesta de los once números publicados entonces (septiembre de 1926 - septiembre-octubre de 1927), sino también las diferencias existentes con lo que hoy se conoce como su primera época, esto es, los seis números aparecidos entre los meses de junio y diciembre de $1925{ }^{4}$

Los integrantes de la empresa en ese segundo momento fueron: además de Atalaya, Giambiagi y Juan A. Ballester Peña, el escultor Luis Falcini, el músico Juan C. Paz y los escritores Álvaro Yunque, Juan Guijarro, Gustavo Riccio, Lizardo Zía y Armando Cascella, nombres a los que eventualmente se sumarían los de Roberto Mariani, Aristóbulo Echegaray y José Salas Subirat y el del crítico de arte Leonardo Estarico. Como vemos, el núcleo inicial era el mismo que había llevado adelante la primer Campana; en aquél entonces se habían sumado Luis E. Soto, César Tiempo -firmando “I.Z.”, por Israel Zeitlin- y Raúl González Tuñón.

En apariencia no hubo fractura entre las dos épocas de La Campana de Palo, percepción que es abonada por los nombres coincidentes y por la adopción de la numeración corrida. Sin embargo y como veremos luego, las diferencias se manifestaron más allá de los cambios producidos en la propia materialidad, visualidad y contenido de la segunda Campana.

\section{PRIMER TIEMPO}

Aunque es natural ocuparnos de establecer los puntos de encuentro y las diferencias entre las dos épocas, también es necesario previamente establecer el nexo con otra publicación anterior. En “Unas cuantas palabras”, nota de presentación y de justificación luego de un “interregno de silencio” que acompañó la reaparición de la Campana en 1926,

\footnotetext{
${ }^{4}$ Los ejemplares correspondientes a ambas Campanas se encuentran localizados en la Fundación Bartolomé Hidalgo para la Literatura Rioplatense. En la Serie Complementaria de Ediciones facsimilares de Capítulo. La historia de la literatura argentina del CEAL (1982), bajo la dirección de Carlos Altamirano y Beatriz Sarlo, se publicaron los números 1 y 4 de la primera época de $L a$ Campana de Palo. Agradezco a Albino Fernández haberme facilitado el volumen encuadernado de la segunda época.
} 
se afirmaba: "El antiguo grupo editor de Acción de Arte -papelucho de grata memoria, pasto para el historiador futuro- se hizo cargo del activo y pasivo de esta empresa con bienes raíces en la tierra de Utopía. Sólo se intenta enlazar el recuerdo de la pasada labor, con la presente. Somos y hemos sido siempre los mismos. Unos cuantos artistas plásticos y algunos escritores” (1).

Con estas palabras se establecía no sólo su propia genealogía sino que, al mismo tiempo y conscientes del valor de su obra, se daban las pistas para escribir su historia. Pero además la mención de Acción de Arte (1920-1922) ${ }^{5}$ permite establecer una nueva cadena de asociaciones y en este punto es importante saber que en 1925 La Campana de Palo retomó el "estribillo" que había servido para autodefinir al grupo inicial reemplazando su título, Acción de Arte, por el de la nueva publicación, La Campana de Palo.

\begin{abstract}
ACCIÓN DE ARTE
no es el vocero de una capilla literaria o artística. ACCIÓN DE ARTE

tampoco es el portavoz de una camarilla de desocupados, entregados a la invención de nuevas teorías o fumisterías de arte; enfermos de notoriedad.

ACCIÓN DE ARTE

es la tribuna de todos aquellos escritores y artistas que desean expresar sin recato su pensamiento; que no tienen intereses creados, y creen que intentar decir la verdad no puede constituir una ofensa para nadie. No pretenden tampoco ser “ORIGINALES”, ni inventar nada nuevo; sólo anhelan depurarse y depurar el ambiente artístico. A nuestros camaradas sólo le pedimos un poco de talento, mucha sinceridad y una gran honestidad. (Acción de Arte 4)
\end{abstract}

En Acción de Arte aparecieron reunidos por primera vez los nombres de Atalaya, Falcini, Giambiagi, Paz, Cascella, agregándose los de los escultores Antonio Sibellino y Nicolás Lamanna, el del pintor Domingo Viau y otros pocos escritores como Álvaro Yunque, Pablo Rojas Paz y Pílades Orestes Dezeo.

Los elementos señalados hasta aquí permiten pensar la segunda época de $L a$ Campana de Palo como un tercer tiempo que encuentra su origen en ese "papelucho", significativamente denominado Acción de Arte. Primer tiempo de definiciones, de encuentro entre los principales actores que se continuó en las dos Campanas y que, sin lugar a dudas, complejiza aun más nuestra aproximación al periódico de Bellas Artes y Polémica.

Luego de establecida la línea de análisis falta responder a otro punto: ¿quiénes fueron los actores aludidos en cada momento? Y si la respuesta es más o menos certera en tanto es claro que Atalaya y Carlos Giambiagi fueron quienes absorbieron las mayores responsabilidades, sin embargo, es necesario aclarar algunos puntos al respecto. En el

\footnotetext{
${ }^{5}$ Hasta donde sabemos hoy resulta imposible hallar una colección completa de Acción de Arte (abril de 1920-1922). Los números que hemos localizado -4, 8, 15, 16, 17, 19, 20 y 22- se encuentran en la Biblioteca del Círculo de Bellas Artes de Montevideo, institución a la que estuvo ligado el escultor argentino Luis Falcini, otro de los participantes de las empresas aquí estudiadas. En Buenos Aires, la Fundación Bartolomé Hidalgo para la Literatura Rioplatense conserva el n. 18.
} 
estudio dedicado a La Campana de Palo en su primera época por Nilda Díaz (358-68), si bien la autora reconoce los nombres mencionados, no se detiene en ellos. Asimismo, la cantidad de seudónimos e iniciales que aparecen, tampoco llaman su atención: "B. Encina” debe leerse “bencina”, C.G. es Carlos Giambiagi, Tristán de Kareol es Atalaya y, probablemente, las restantes iniciales empleadas oculten a los mismo actores. Por otra parte, Juan Antonio Ballester Peña (1895-1978), ilustrador de la mayoría de sus números e integrante del grupo editor, tampoco es mencionado. Esto tal vez se deba al hecho que los tres actores parecen pertenecer en primera instancia a un ámbito ajeno al de las letras, lugar desde donde es abordada la revista.

En realidad lo que hace Díaz es poner especial énfasis en la situación de la revista en relación con los dos grupos claramente identificados a mediados de los años '20: el Grupo de Boedo y aquél nucleado en torno al periódico Martín Fierro y aunque esta contextualización pueda resultar incompleta, sin embargo ella acierta al intuir el espacio en el que se ubica esta primer Campana dentro del "modelo anarquista, teñido con una buena dosis de utopía social-humanitaria” (366).

Por otra parte, en el trabajo dedicado al Suplemento Semanal de La Protesta, aun cuando Lidia Maroziuk escribe desde el ámbito específico de la historia del arte, no manifiesta la intención de identificar a quienes llevaron adelante ese emprendimiento (205-16). Y en este punto el saber que "At.” es Atalaya, que es Giambiagi quien se oculta bajo el seudómino de “Zero” -en la Campana será “el hombre de la selva”- o que "R.S." es Ret Sellawaj, seudónimo de Ballester Peña no parecen ser datos menores.

Pero lo que es más importante de destacar aquí, es que detrás del problema de identificación enunciado se esconde en realidad otra cuestión no menos importante que hace a la ideología política misma de estos intelectuales. Si en el caso de Arístides Gandolfi Herrero, uno de los escritores más estrechamente ligados a la Campana en sus dos épocas, su seudónimo “Álvaro Yunque” es aquel por el que tanto entonces como hoy en día se lo reconoce sin ofrecer ningún inconveniente, en el caso particular de los otros actores mencionados la situación parece ser otra.

El uso y aun el abuso-como en el caso de Atalaya-de seudónimos, lleva necesariamente al efectivo ocultamiento de la identidad. Es en el optar por ese nuevo tipo de anonimato donde reside la concepción del propio trabajo. No se trata de ser crítico de arte, escritor o artista en los términos que estas profesiones se encuentran claramente diferenciadas en el campo cultural argentino de los años veinte y proporcionan un determinado estatus en la sociedad a quien los ostenta, sino de ser "obreros de arte”. La máxima de Atalaya "un artista no vale más que un picapedrero” (AA. Textos programáticos, 5) encuentra su explicación en la plena convicción de que:

somos simples artesanos que lograremos con constancia, trabajo, y afán, a realizar obras que tendrán su encanto por la honestidad que pongamos en ellas. Pero no somos artistas, ni tenemos derecho a escribir y pintar en artistas. Rebajemos nuestras pretensiones, limitemos nuestra visión, apliquémonos a cosas humildes y confesemos que aún no podemos aspirar a ser artistas. Basta de obras pedantes, de pretensiones desmesuradas y de pobre realización. (AA. Textos programáticos, 2) 
Estos actores carecían de una educación formal y trabajaban para poder alcanzarla, pero no para ubicarse por encima de los demás sino todo lo contrario, para contar con las herramientas necesarias para educar al proletariado. Como lo afirma Lily Litvak: "en la síntesis estética-política-social que los anarquistas llevan a cabo pretenden destruir el status de la obra de arte como goce privativo de las clases pudientes y como producto exclusivo de artistas profesionales. Intentan otorgar el derecho de gozar y de crear obras artísticas a todo individuo, volviendo al arte a sus raíces populares” (10).

Fue en Acción de Arte donde estos objetivos aparecieron formulados con mayor claridad. El mismo título elegido para la revista remitía al principio anarquista de la acción directa y, en uno de los momentos más difíciles de la historia del anarquismo en la Argentina, ${ }^{6}$ el grupo de jóvenes que la constituían estaban plenamente convencidos de que sólo del actuar por sí mismos se alcanzaría la liberación de los trabajadores del yugo esclavizante del Estado. Desde los años de los anarquistas organizadores (1890-1905), el principio táctico de la acción directa estimuló, como lo afirma Golluscio de Montoya "la aparición de la variada serie de manifestaciones constructivas, tan característica de las formaciones libertarias (fundación de grupos anarquistas, actividades docentes, culturales, literarias, periodísticas, etc.)” (49-50) y al iniciarse la nueva década estaba lejos de agotarse.

En el caso de Acción de Arte, el actuar desde el arte llevó a propiciar actividades que fueron promocionadas como las conferencias pedagógicas ("El placer estético” o “El arte y la moral”), la inserción en sus mismas páginas de los denominados folletines que podían enseñar el procedimiento para hacer inalterable la acuarela, los rudimentos básicos de la pintura o sobre la cualidad y el comportamiento del color. Otro punto recurrente fue la crítica a las instituciones oficiales -Museo, Academia, Comisión y Salón Nacional de Bellas Artes- en tanto representaban los mecanismos del Estado en pleno funcionamiento al iniciarse la década del veinte. Era el llamado “gobierno de las Bellas Artes” que con su dirigismo marcaba los rumbos a seguir, dictaminaba qué era bueno y qué malo, premiaba y castigaba y ejercía acciones coercitivas que iban en contra de la libertad individual. Frente a la necesidad de reformas, el grupo de Acción de Arte promovió un Salón Independiente y la creación de una Asociación Independiente de Artistas Plásticos, propició encuestas sobre los salones que debían "ser libres como plazas” y acerca de la necesidad o no de reorganización de la Academia. ${ }^{7}$

${ }^{6}$ No hemos podido consultar el artículo de Osvaldo Bayer "El anarquismo y la década del veinte" (Cuadernos de historia. 1 Vertientes del nacionalismo revolucionario. Buenos Aires: Liberarte ediciones, s.d., 51-68), que suponemos importante dado que la mayoría de los estudios dedicados al anarquismo en Argentina cubren hasta mediados de los años '10. En el caso particular de La Protesta, durante el período marcado por la Semana Trágica en 1919 y los sucesos del año 1921 en Entre Ríos, Chaco y la Patagonia, el diario sufrió secuestros, clausuras y prisión de muchos de sus redactores, apareciendo algunas de sus ediciones en la clandestinidad y en ciertos momentos siendo suplantada por otros diarios comprometidos con el movimiento obrero. Cf. los trabajos citados en nota 3.

${ }^{7}$ Años antes el Grupo de Barracas, luego conocido como Artistas del Pueblo, también de filiación anarquista, realizó las primeras acciones en contra del orden regulador oficial: en 1914 organizó el Salón de Rechazados y en 1918 el Salón de Independientes. Sin Jurados y Sin Premios además de crear la Sociedad Nacional de Artistas. Sobre este grupo, cf. Muñoz 116-30. 
Sin embargo, todos y cada uno de estos cuestionamientos aparecían filtrados por ese tono de humor que habría de distinguir luego a las dos Campanas; la consigna era castigat ridendo mores: “corrige las costumbres riendo".

SEGUNDO TIEMPO

A pesar de que no tenemos noticias acerca de las causas que determinaron la desaparición de Acción de Arte, sí sabemos que fue más o menos contemporánea al lanzamiento del Suplemento Semanal de La Protesta. El objetivo del "cotidiano" al publicar un suplemento había sido el de completar su labor pues entendía que había algunos aspectos que eran descuidados o relegados a un segundo plano acuciado como estaba por la necesidad de dar cuenta del movimiento obrero con material informativo. El suplemento permitiría completar su obra de manera más eficaz al cumplir con sus objetivos doctrinarios y ocuparse de los problemas de orden internacional del anarquismo, particularmente en lo referido a la actualidad rusa y al giro producido en su desenvolvimiento al iniciarse la nueva década. En este sentido, la propaganda realizada por los bolcheviques, que presentaba a los anarquistas como contrarrevolucionarios, obligaba a mantener lo que denominaba una "guerrilla contrarrevolucionaria” ("Nuestros objetivos” 1).

El primero en incorporarse al Suplemento fue Atalaya, cuyas notas aparecieron alternativamente en el cuerpo del diario y en el semanario; le seguirían luego Giambiagi y Ballester Peña, aunque sólo durante 1925 la acción de este trío cobraría mayor coherencia. ${ }^{8}$ A comienzos de ese año Atalaya había ya expresado su interés en fundar una nueva revista de la que participaran los antiguos integrantes de Acción, que se llamaría Artes Plásticas y probablemente haya sido la falta de medios económicos la que impidió su lanzamiento. Esta misma razón es la que debe haber determinado que fuera La Protesta la encargada de financiar una nueva publicación, la primer Campana que no obstante contaba con unos pocos anunciantes, entre ellos la Casa Iturrat de la familia del mismo Giambiagi. Un antecedente para este hecho en apariencia inusual puede encontrarse en la misma Martín Fierro (1904-1905) de Alberto Ghiraldo -en esos años al frente de La Protesta- que a partir de su número 31 fue anunciada como suplemento del diario.

De esa manera, más que una revista dedicada exclusivamente a las artes plásticas, lo que apareció fue una revista de perfil cultural; para el nuevo grupo editor no ha de haber resultado difícil adecuarse a un posible requerimiento en ese sentido en tanto los lazos con otros actores del campo intelectual eran fuertes, en particular con Álvaro Yunque que también trabajaba en la redacción de La Protesta y, a través suyo, con otros escritores que actuarían como colaboradores de la Campana.

El primer número de la nueva publicación apareció en el mes de junio de 1925, con el formato cuadernillo de 32 páginas -sólo el n. 3 llevaría 30- editada en papel prensa. Desde su título -con las mismas letras “desaliñadas” elegidas para Acción-se anunciaba

\footnotetext{
${ }^{8}$ Gravemente enfermo, desde mediados de 1923 a julio del año siguiente, Atalaya debió internarse en una clínica en La Punta (Perú). Durante ese tiempo, fue Giambiagi quien ocupó su lugar en el Suplemento. No obstante, algunos de sus artículos sobre la situación política peruana fueron conocidos en el cuerpo del diario durante el año 1924.
} 
cuál era el camino a seguir: en un lenguaje "metafórico" se deslindaba cualquier asociación con el poema de José Hernández, a la que aquél parecía aludir.

En su trabajo ya citado, Nilda Díaz se ha ocupado de marcar la fuerte connotación del título elegido, citando el poema de José Hernández: "Para él son los calabozos,/para él las duras prisiones;/ en su boca no hay razones/ aunque la razón le sobre;/ que son campanas de palo/ las razones de los pobres” (360-61). El rechazo explícito que descubre en las palabras iniciales "La campana de palo... Y las razones de los pobres... No. No es eso. A pies juntos saltaremos por encima del refranero criollo y de los cantares anónimos” (Atalaya "Las Campanas” 3, reproducido en Schwartz 257-59) puede entenderse por la misma posición de Atalaya al analizar dos “poemas nacionales”-Briznas de hierba [i.e. Hojas de hierba] de Walt Whitman y Martín Fierro de José Hernández. En “Nihilismo” -un texto sin fecha pero que debe ser situado ya avanzada la década del veinte- el ponía sobre el papel qué era lo que diferenciaba a ambas obras. Mientras que la sustancia y contenido del primero era "el ensayo generoso de la virgen experiencia de una moralidad nueva, desnuda, arrojada, libre de todo caduco prejuicio”, en el segundo su contenido era

la vetusta experiencia de un hombre viejo quien nos da consejos para quedar bien con el juez, y tener siempre a mano un palenque, donde podamos rascarnos y apoyarnos en los casos de apuro. Moral de viejo, moral retrógrada y nefasta para la juventud; moral acomodaticia que no dejamos de practicar un solo día, que encaja tan admirablemente con nuestra vieja haraganería sentimental. Como se ve, hasta uno de los protagonistas de nuestro mejor poema del terruño, hubo de ser un viejo con argucias y razones de viejo.

(AA. Reflexiones, 1)

En realidad, lo que la Campana hacía desde su título era alzarse en contra de la situación en la que el periodismo moderno se hallaba varado; la "misérrima” campana de palo venía a recuperar la función social que las campanas cumplían durante el medioevo, asociación que no resulta extraña en el contexto que venimos analizando:

Izadas en la magnitud del cielo, unas campanas cantarinas y alegres, como hechas de plata; otras gravemente profundas y broncas, como forjadas en maleable hierro, colgaban de la torre [sic] de las más altas torres del villorrio, de la ciudad o de la metrópoli, y eran todas ellas, con la lengua de sus badajos, las múltiples lenguas y el verdadero idioma del pueblo, de la multitud, de la prole metropolitana elegante o astrosa. Ellas doblaban, oraban, cantaban o furiosamente como Gorgonas desmelenadas tañían a rebato, llamando a somatén. Esto era en el antiguo tiempo. No aparecida aún la babélica invención del cotidiano papel impreso insumían en sí las funciones de un periodismo rudimentario, sonoro y vibrante, eucarístico y regocijado. (Atalaya "Las Campanas” 3)

Pero ¿dónde sonaba la Campana? Una de las litografías empleadas a modo de viñeta la muestra de dimensiones colosales pendiendo de una grúa frente a fábricas humeantes; otro linóleo empleado como bandeau la representa “alada” sobrevolando la gran urbe y multiplicando su sonido en cientos de aves que cubren su cielo; ellas llaman a un despertar de la conciencia. El búho en la noche, sobre una rama de la que cuelga la campana, con un fondo de luna que ha de identificar el sello editor, marca tanto la soledad en que ellos se encontraban como su capacidad para ver incluso en tiempos “oscuros”. 
Fue en sus números 4, 5 y 6 donde la revalorización del grabado alcanzó gran fuerza y coherencia en tanto el discurso visual remitía directamente a los postulados que dieron vida a la revista. Desde la cubierta la figura del hombre adquiere tamaño monumental, destacándose el esfuerzo del trabajo que implica sea cargar o tañer la campana, hasta que finalmente el objeto en sí desaparece. Anónimos, en su mayoría los grabados empleados como ornamentación de página fueron realizados por Ballester Peña y otros pocos por Giambiagi. Su inclusión en la revista no sólo tiene que ver con la facilidad que la técnica del grabado ofrecía a los efectos de su reproducción sino también con el denominado renacimiento del gravure sur bois originale que tuvo lugar a fines del siglo xIx proyectándose al siguiente y que ocupó a artistas como Gauguin, Félix Valloton, los expresionistas alemanes, Käthe Kollwitz, el grupo Les Cinq y Frans Masereel, entre muchos otros.

Pero además, dentro de la estética anarquista al grabado se le asignó históricamente una misión social en tanto "la imagen difundida masivamente perdía su calidad aristocrática de ejemplar único a tirada limitada, y venía a disputar su lugar al texto como instrumento único de propaganda ideológica y cultural” (Litvak 61).

$\mathrm{Al}$ mismo tiempo y en lo que hace a los artistas y al crítico de arte que integraban el grupo editor de la Campana, el carácter anónimo, tenía que ver con la propia concepción del trabajo que hemos explicado antes. En parte era respuesta a esa pregunta acuciante: ¿cómo vivir honradamente del arte? En carta a Atalaya, fechada el 22 de septiembre de 1921, Giambiagi fijaba su posición:

[...] el cuadro de caballete y otras sonceras, son arte chic -para burgueses-. La decoración solamente daría -quizás- un medio de vida. Pero la decoración se hace para la burguesía y ésta exige un arte falaz y mentido. Cabe sin embargo decorar libros y ensayar una decoración escultórica honrada. Está el grabado -módico y difusible- las viñetas, los affiches... Todo es posible si a pesar de todo, trabajáramos arte, como jornaleros. [...] No más exposición de cuadros, sino de trabajos. Una fuente, un pilar, una reja, un almohadón, una tapa de libro, etc., anónimo[...] (212-13)

Se trataba de romper con todas las jerarquías aceptadas, esto es, la división entre artes mayores y artes menores. En septiembre de 1924, en un artículo titulado "La decoración del libro", At. afirmaba: "Debemos, pues, pensar que no hay tarea ruin, ni pequeña, ni desmedrada, cuando se la ejecuta con amor y pujanza. Por infinidad de caminos se va a la meca del arte y éste, el de la decoración del libro, de la revista o del periódico, no es de los menores. Talentos menores serán los que lo ejercen hoy, profesionalmente, ya que todos los aspectos de la actividad humana son susceptibles de lograr la sublimidad” (AA. Álbum Recortes, 5).

En relación con su contenido, esta primera Campana dio un espacio significativo a la labor doctrinal y pedagógica que se filtraba de diversas maneras en sus páginas, no sólo en las voces de Rafael Barret o Max Nextlau, sino también en cada una de las secciones fijas de la revista: “Cuentos exóticos”, "Música y musicantes”, “Arte plástico y anexo”, "Miscelánea de expositores y salones”, "Las máscaras teatrales”, "Escaparate literario”, "Retratos de ayer y de hoy".

Pero volviendo al punto que nos interesa remarcar, existen varias pistas que nos llevan a afirmar la estrecha relación entre La Campana de Palo y La Protesta que exceden 
el temprano encuentro de Atalaya con algunos de los principales actores del movimiento anarquista en torno a los años ' $10 .^{9}$ La dirección de la revista era Perú 1533 , mientras que la del diario Perú 1537; Mariano Torrente-administrador del diario entre 1917-19 y luego regente de la imprenta- era mencionado por Atalaya en su correspondencia al momento de tomar decisiones clave relativas a la vida de la revista ${ }^{10} \mathrm{y}$ lo que no es menos importante, los dos primeros libros de la Biblioteca de la Editorial La Campana de Palo-Zancadillas de Yunque y Un poeta en la ciudad de Gustavo Riccio- fueron impresos en los Talleres Gráficos del diario. Es precisamente la labor editorial de la revista la que aporta mayores datos. Atalaya, él mismo escritor, le confiaba -en carta fechada el 17 de diciembre de 1924- a su amigo Falcini:

También Carlo, me incita a componer un libro de cuentos, decorados por él.- Por qué no confesarlo, es una gran tentación, todas estas incitaciones; pero temo que después me haya de arrepentir amargamente. Mas supongamos, que estuviera satisfecho, se presenta la dificultad del editor.- Hablas de la editorial de la Campana, sin pensar que a Yunque, si le publican es por ser una firma cotizada en el mercado literario.- Discutido o no, es un valor innegable, y yo que conozco personalmente, declaro que es el único muchacho con un gran porvenir literario. [énfasis agregado] (MNBA/32)

¿Cuál pudo ser el interés del diario en financiar este tipo de ediciones? En la nota de El Grupo Editor de La Campana que acompañó la aparición de Zancadillas se afirmaba que la selección de autores debía ser rigurosa en tanto las obras a publicar debían adecuarse a la "modalidad social” del propio trabajo, esto es "Usar la pluma a modo de arado". ${ }^{11} \mathrm{Y}$ todavía, al presentar el libro de Riccio se aclaraba: "creemos que contribuimos a colaborar en la obra realista y renovadora que está realizando entre nosotros un grupo de poetas jóvenes, al hallar motivos para sus poemas en la afiebrada multaneidad de la urbe y en la dolorosa tragedia cotidiana de sus semejantes" (“Nota”, Riccio 95).

En lo que concierne a la Editorial, sabemos que entre 1904 y 1932 publicó por lo menos 40 títulos, la mayoría de ellos lanzados durante los años veinte a veces a través de distintas series que no siempre tuvieron continuidad en el tiempo: Biblioteca de "La Huelga General”, Biblioteca Tierra y Libertad, Tiempos Nuevos, Biblioteca La Protesta, Biblioteca de El Productor, Propaganda emancipadora entre las mujeres, Pensadores y

\footnotetext{
${ }^{9}$ Conviene tener presente aquí que la actividad literaria de Atalaya en Buenos Aires, luego de sus primeros trabajos publicados en la ciudad de Rosario, tuvo lugar en Ideas y Figuras de Alberto Ghiraldo. Allí apareció su primera nota de arte dedicada al Salón Nacional de Bellas Artes de 1912 y se publicó también uno de sus cuentos bajo el seudónimo Alfredo Valenti, en 1913. En el Archivo Atalaya existe, asimismo, una carta dirigida a Eduardo G. Gilimón -sucesor de Ghiraldo al frente de La Protesta- probablemente de mediados de los años 10.

${ }^{10}$ En carta datada el 17 de diciembre d 1925, At. le informaba a Falcini acerca de los atrasos de la revista y le decía: "Hace próximamente un mes que está armada y impresa la tapa y los pliegos de adentro. Y así estamos. En una reunión que tuvimos ayer, es decir yo, Ballester, y Torrente, quedó decidido, que aparecido el sexto número, se escribiera una noticia, expresando que por el escaso apoyo, saldríamos cuando pudiéramos” (MNBA/30).

${ }^{11}$ El Grupo Editor de La Campana de Palo. S/t. (Yunque, 1926 s/p).
} 
propagandistas del anarquismo. Ellas reunieron las principales obras del anarquismo doctrinario, con ensayos históricos y reivindicadores del movimiento obrero a nivel internacional y nacional: Miguel Bakunin, Errico Malatesta, Rudolf Rocker, Eliseo Reclus, Luigi Fabbri, Max Nextlau, Sébastien Faure, William Morris, Petr Kropotkin, Johann Most, Pierre Quiroule y el mismo Abad de Santillán, entre muchos otros. ${ }^{12}$

Uno puede pensar que así como el Suplemento Semanal vino a completar la labor del diario, el apoyo financiero para el emprendimiento de La Campana de Palo debe haber estado relacionado con la misma necesidad: la revista y su Biblioteca vendrían a ampliar el espectro pedagógico de su obra, tal como contemporáneamente lo hacía con gran éxito la Cooperativa Editorial Claridad al transformar Los Pensadores (1924-1926) en revista y lanzar en 1924 la serie Los Nuevos -con Gustavo Riccio, Elías Castelnuovo y Antonio Zamora como directores- colección en la que se conoció Versos de la calle de Álvaro Yunque.

Fue precisamente Yunque quien por primera vez estableció el vínculo que venimos estudiando; muchos años después, él recordaba que: "La Campana de Palo, siempre teniendo por animador a Atalaya y Giambiagi coincide también con la presencia del Suplemento Semanal de La Protesta, donde uno y otro yugan con denuedo [...] La Campana de Palo y el Suplemento, continuaron la línea de agresividad, ansias de hacer justicia y descabezar falsos ídolos que tuvo Acción de Arte.” (“Carlos Giambiagi” 110). Testimonio ampliado posteriormente al afirmar abiertamente que la Campana era publicada por La Protesta (Pelletieri “El peor libro de 1926” 2).

De esta manera, los grabados de Ballester Peña y, en menor medida, los de Giambiagi no sólo ilustraron la Campana sino que construyeron la visualidad del Suplemento. Al mismo tiempo, las notas de arte de At. pueden ser leídas en un tandem: a veces en la revista sólo aparecía la noticia de una exposición y el artículo en el semanario o viceversa, se trataba de una doble tribuna que buscaba aprovecharse al máximo. ${ }^{13}$

Sin embargo existieron algunas problemáticas que marcaron seguramente un punto de roce con los compañeros anarquistas. En particular, las frecuentes objeciones del crítico a los artistas más conservadores y probablemente su interés en aquellos representantes de la vanguardia porteña -como por ej. Xul Solar, Emilio Pettoruti y Norah Borges- y por las tendencias internacionales más modernas como el grupo Novecento Italiano.

De hecho, en la Campana el rechazo a la vanguardia se propuso desde las letras mientras que las expresiones de renovación plástica estuvieron ausentes de sus páginas. ¿Por qué poner en riesgo el apoyo económico que podía tener la revista si de todas maneras en el Suplemento tenía margen para expresar sus opiniones personales que generalmente se filtraban en las extensas notas dedicadas al Salón oficial o a las exposiciones colectivas?

\footnotetext{
${ }^{12}$ Esta información ha sido elaborada a partir del catálogo online de las colecciones del International Institute of Social History (IISH), Amsterdam. (http://www.iisg.nl/index.html)

${ }^{13}$ Refiriéndose al Salón Nacional, en carta fechada el 24 de septiembre de 1925, Atalaya le decía al escultor: "Tú me sugieres formular una encuesta. Poseo, tan poca autoridad, que temo poco o nadie contesten. De todos modos he de probar. En la Campana y el Suplemento, he de continuar la crítica. En el primer número de la Campana fue imposible, por hallarse listo el material” (MNBA/16). En la misma Correspondencia aparecen varios comentarios de tono similar.
} 
Este punto es de particular relevancia ya que desde el anarquismo y con su rechazo a toda norma que limitara la libertad de creación del artista, parecía poco probable que existiera una oposición a la vanguardia. Sin embargo, históricamente la fórmula con que habitualmente se la objetaba -el arte por el arte- predeterminaba cualquier lectura de sus obras en tanto lo que debía primar era el mensaje sobre la forma.

Es de particular relevancia recordar, como lo señalan Andreau, Fraysse y Galluscio de Montoya, que: "Resulta asombroso comprobar que los escritores anarquistas tan revolucionarios en cuanto a la visión del mundo, hayan sido tan poco innovadores en lo que concierne a la literatura. Existe un defasaje impresionante entre el contenido radicalmente subversivo de los textos y la forma convencional que les es dada y que adhiere, en sus grandes líneas, a la retórica tradicional del arte burgués contemporáneo” (13). Un arte realista, con un claro mensaje de denuncia, accesible al pueblo era el que habían sustentado los Artistas del Pueblo -Adolfo Bellocq, Abraham Vigo, José Arato, Agustín Riganelli y Guillermo Facio Hebequer- que en su accionar como grupo, estuvieron ligados a Boedo, colaborando con Claridad e ilustrando varios de los libros de los autores ligados a ella.

At. manifestó su desacuerdo respecto de sus obras pues reconocía en ellas una imposibilidad para alcanzar un arte colectivo y popular. Refiriéndose a Arato, afirmaba que su pintura quedaba

\begin{abstract}
en el aspecto de una pintura de intención popular, apresando y contentándose con lo pintoresco de la humildad, de la pobreza etc. El intento de trasladar el arrabal, por la peregrina coloración de su infortunio, vivaz y sombrío, que gime, canta, se adolora, ama y mata, refundiéndolo en la literatura, en la música y artes plásticas, ha sido ya realizado numerosas veces con suerte incierta y diversa. El conventillo y sus adyacencias fue exprimido hasta el bagazo, y proporcionó infinitos temas. (“Exposición José Arato” 244)
\end{abstract}

Para él estaban mucho más cerca de alcanzar una verdad de tipo colectivo

\begin{abstract}
las tendencias artísticas avanzadas -cubismo, etc.- que se inclinan a lo impersonal, al anonimato de los primitivos artesanos -quienes desaparecían tras de sus obras individuales para integrarse en un esfuerzo común. Y ellas, en sus abstractas geometrizaciones, es más probable que algún día se conviertan en escritura artística, colectiva y popular, que quienes en su instinto y buenas intenciones se ponen graves y trascendentalizan humanitariamente, e intentan estilizar haciendo preciosismo y verismo en confusión babélica. Hay un resto de arte burgués -por provincianismo- en el fondo y en la superficie de sus procedimientos, del cual no se atreven a despojarse. [énfasis agregado] (“Exposición” 244-45)
\end{abstract}

En el caso de este crítico de arte y en relación con su lectura de las propuestas renovadoras del arte a partir de Cézanne, debe recordarse su temprano contacto con el círculo de Alberto Ghiraldo e Ideas y Figuras; no sólo Juan Más y Pi-quien le dedicó un ensayo a Ghiraldo- fue el encargado de difundir - ¡adhiriéndose!- a los postulados del futurismo desde El Diario Español incluso antes que Rubén Darío publicara su "glosa” 
del manifiesto fundacional en La Nación, sino que en la misma revista Filippo T. Marinetti apareció como colaborador. ${ }^{14}$

El interés que reviste la obra crítica de Atalaya reside precisamente en este punto: lejos de haberse visto limitado por las consignas estéticas del anarquismo, eligió el actuar libre de todo prejuicio o convención, por lo menos en lo que se refiere a artes plásticas. Esto se manifestó ya en Acción de Arte donde se incluyeron notas sobre "El expresionismo: la joven poesía” de Pol Michel (2) y sobre los ballets rusos, esta última de Jacques Liptchiz (4) y, entre los folletines de difusión, un "Estudio sobre el dadaísmo" firmado por Albert Gleizes (4).

Era su postura respecto de lo que el arte debía ser para alcanzar un carácter colectivo y anónimo que, en el contexto anarquista de los años veinte, provocó el rechazo en sus compañeros ácratas. Y en algún punto esto debe haberlo obligado a cierto tipo de adecuación a sus requerimientos. En un texto sin datar, pero que debe situarse a mediados de los años veinte, Atalaya explicaba cuál era su concepción de la novela. Él aclaraba allí su posición y, al intentar abordar la problemática de la prostitución, reflexionaba consciente de su propia contradicción:

Una novela corta con el fin de presentar el carácter altamente grotesco y repugnante de la actual prostitución, organizada en casas de lenocinio. Para mostrarle en toda su vil crudeza he de servirme de alguna especie animal, a los que les haré animar la existencia de los prostitutos y prostitutas.

¿Cuál animales elegir? Allí se halla el punto más difícil. No me inclino ni por los perros, ni por los gatos, ni ningún otro animal que se avecina a la especie humana en algunos de sus aspectos más vitales. No sé si me decido por las vacas y los toros. El contraste debe ser lo más grotesco, lo más monstruoso.

En fin, poseo una nebulosa idea que busca demostrar cuán lejos, cuán lejos se halla especialmente la presente prostitución que sostiene a todos los estados del mundo, de lo que podría ser el amor libre entre los dos sexos.

Yo como Giambiagi, que disentía con el impresionismo, fui de la escuela realista y racionalista a pesar mío. Siento mejor la novela fantástica y hasta simbólica que la novela realista de una apariencia puramente física. Soy un pésimo observador y aun más pésimo memorista. Mis reflexiones se alimentan de subjetivismo que extraigo de [mí] mismo, con una absoluta prescindencia de la realidad cotidiana.

Sin embargo, tan encallecido estoy de un realismo chiquito, que quizás me haga cultivar los dos géneros, a pesar mío. (AA. Reflexiones, 57)

${ }^{14}$ Cf. F. T. Marinetti. “Gabriel D’Annunzio”. Ideas y Figuras. Buenos Aires, 2.27 (2 marzo 1910), s.p. En la nota editorial que acompañó este artículo, luego de ser presentado con palabras laudatorias referidas a su obra literaria, Ghiraldo explicaba que el "Futurismo, tendencia de arte por él planeada y que ha merecido ya la más unánime discusión en todo el mundo civilizado, proclama una renovación de todas las ideas, y, sobre todo, la afirmación de que si cada época debe tener una característica en el arte como en la vida, la nuestra debe producirla cuanto antes, siendo necesario, para ello, que olvide las enseñanzas de las generaciones pasadas y que se cree su ética y su estética." [Alberto Ghiraldo]. "F. T. Marinetti”, loc. cit. Sobre la recepción del Manifiesto Fundacional del Futurismo, véase Artundo: “El futurismo en Buenos Aires: 1909-1914”. 
Uno puede pensar que es esto lo que determinó su ubicación particular en el campo intelectual de mediados de la década del veinte, imposibilitado como lo estaba por su propia ideología de alinearse junto a cualesquiera de los grupos que radicalizaban sus posiciones. Por un lado, hacia mediados de 1925 los martinfierristas habían alcanzado una imagen pública coherente con sus objetivos de renovación, mientras que el Grupo de Boedo, reunido ahora en Los Pensadores pugnaba por conformar un "frente único de la mentalidad izquierdista” que sólo se concretaría a fines de ese mismo año.

Resulta evidente, además, que los movimientos de los actores en el campo y los deslizamientos que se producían entre la Campana y Los Pensadores lo tenían a Yunque como pivote. En este sentido, el caso de Luis E. Soto es ejemplar: comenzó su actividad en Inicial. Revista de la nueva generación, escindido ese grupo pasó rápidamente por Proa con una nota sobre Versos de la calle para luego incorporarse a Renovación y a Los Pensadores llegando durante 1925 a cumplir un papel rector en la orientación de esta última revista, al tiempo que colaboraba con la Campana.

Sin embargo, al considerar esta publicación en su conjunto no es la problemática Boedo-Florida la que ocupa el lugar central aunque esté presente. Lo que se observa en La Campana de Palo es la voluntad de alcanzar un vehículo de expresión propio; sus seis números manifiestan unidad y coherencia y sus intereses -política, literatura, arte, música- comparten equilibradamente su espacio. Las causas que determinaron su desaparición en diciembre de 1925 fueron principalmente las económicas -y éstas claramente relacionadas con el escaso número de lectores con el que contó- ${ }^{15}$ y la imposibilidad material de sus redactores para dedicarle el tiempo necesario. No obstante ello, la labor siguió con su Biblioteca que editó los libros de Yunque y Riccio entre marzo y julio de 1926. Este fue el mes en el que At. publicó su última nota original en el Suplemento Semanal previo su quiebre definitivo con La Protesta.

TERCER TIEMPO

Uno de los aspectos que más llama la atención durante la década del veinte es la proliferación de revistas que se constata y esto es más notorio si comparamos su número en relación con las aparecidas durante el decenio anterior. ${ }^{16}$ Los títulos se suceden unos a otros y lo que no es menos importante, dentro de cada revista, la propia dinámica imprime cambios notables. Al tiempo que Los Pensadores se transforma en Claridad, Nosotros adopta nuevas estrategias que le aseguran su permanencia en el tiempo.

En el caso de la Campana los cambios son profundos. No sólo se trata del alejamiento traumático de At. del diario anarquista y su ruptura con el movimiento que le habría de

\footnotetext{
${ }^{15}$ El 5 de agosto de 1925, a dos meses de su aparición, Atalaya le confiaba a su amigo Falcini: "Sin desmentir, los cálculos, que yo había hecho sobre esta publicación, hasta ahora la aceptación ha sido casi nula. Es algo previsto por mí, aunque la realidad supere lo que pude imaginarme. No disimulo, que esto me duele profundamente. Da también el caso que La Protesta se halla en una malísima situación financiera, lo que contribuye a agravarlo todo. Giambiagi, me escribió que en San Ignacio, no encontró La Campana, ni un lector” (MNBA/26).

${ }^{16} \mathrm{Al}$ respecto, véase Pereyra, La prensa literaria argentina (1995). Es el registro más importante y actualizado dedicado a revistas argentinas.
} 
permitir una libertad mayor de expresión, sino que además la experiencia editorial que sumaba el grupo editor se sintió en la introducción de cambios sustanciales. En primer lugar, su formato. En el abandono del cuadernillo y la elección de otro muy próximo al del mismo Suplemento lo que se buscaba a través de sus ocho páginas era una mayor agilidad e inmediatez en el contacto con sus lectores, objetivo al que no era ajena la propia experiencia de Martín Fierro. Las letras de su título siguieron con ese desaliño que caracterizó a Acción de Arte y la primera época de la Campana y su propio movimiento en el plano volvía a subrayar su espíritu cantarín y el castigat ridendo mores que también las había distinguido

Algunos elementos iconográficos reaparecieron -como la campana entre las fábricaspero también se emplearon otros nuevos más sintéticos; ahora es una viñeta la que se mueve en las páginas sin ocupar un lugar definido: una mesa de trabajo iluminada por una lámpara y sobre ella la campana y a su lado un martillo, casi la misma imagen que años después y con algunas variaciones identificaría a la revista Conducta (1938-1943) de Leonidas Barletta.

Su subtítulo -Periódico mensual. Bellas Artes y Polémica-marca el reconocimiento de los tiempos necesarios para concretar un número. Subraya también el lugar que ha de ocupar el arte en sus páginas aunque el agregado del término general "polémica” indica que este periódico no se lanza a sí mismo desde la especifidad de una publicación de arte, sino que desde su punto de partida elige una posición combativa que ha de expresarse en varios campos.

En la construcción de su propia imagen visual, esta Campana continuó con su opción por el grabado y las xilografías de Giambiagi cobraron una presencia mayor a la par que indican su compromiso más activo con el periódico. Él construyó una pequeña galería de personajes y paisajes; se trata sobre todo del paisaje interior del hombre, en su trabajo cotidiano en el campo o aquél otro que sin posibilidad alguna de salir de esa condición a la que lo condena la sociedad, toca su acordeón y a sus pies tiene el vaso y la botella; unos y otros son dignificados por la mirada afectuosa de otro trabajador.

El grabado mismo se convierte en un valor. Ballester Peña fue el encargado de definir una nueva imagen para el tiraje de 100 acciones cuyo valor nominal era de \$5: en ella reaparece esa campana alada que ocupa dos cuartas partes de la superficie y sobre la que se monta la figura grotesca y amenazante de un hombre que sostiene una antorcha, que contrasta claramente con la tranquilidad del paisaje en el que se inserta. El objetivo del grupo era crear un fondo de reserva para las nuevas publicaciones de la Editorial de La Campana de Palo y esa acción daba derecho a dos folletos “y de yapa le regala un grabado de nuestro insigne grabador que cubre su retirada con el pseudónimo bárbaro Ret Sellawaj[...]”. (“Correo del piccolo navío” 8).

En el nuevo proyecto editorial, anunciado en su número 10 (diciembre 1926) se explicita su "agresividad, nuestra desembozada acometividad, nuestra pasión contra todo lo que nosotros consideramos injusticia, ignominia, venalidad y literario proxenetismo." ("Una aventura editorial” 3) . Con medios económicos ahora más reducidos, los folletos a publicar -literarios y artísticos- sin embargo, no perderían en calidad mientras que su precio -30c- pondría al alcance de todos pensadores, artistas y críticos de orden internacional-Tolstoi, Bernard Shaw, Adolfo Wildt, Emile Bernard-, escritores argentinos 
-Soto y Yunque-y artistas de la talla de los desaparecidos Martín Malharro, Ramón Silva, Nicolás Lamanna y Walter de Navazio.

En el único folleto aparecido -Zogoibi: novela humorística de Luis E. Soto- se concretaron los ideales expuestos tanto porque se trataba de una violenta crítica a la novela de Enrique Larreta a la que el título aludía -éxito editorial del año junto a Don Segundo Sombra de Ricardo Güiraldes- como por sus cuidados detalles. Los grabados de Ret Sellawaj y un tiraje especial: 1.000 ejemplares comunes, 100 para accionistas y 50 para el comercio, numerados de 1 a 100 y de 1 a 150, indicando el número de cada ejemplar en el ex-libris. Esa misma intención de superar los escollos que la falta de medios podría producir sobre la calidad del objeto fueron también tratados con el periódico mismo, anunciando un tiraje especial de 50 ejemplares numerados, de la misma manera que lo hacía contemporáneamente el periódico Martín Fierro con el que compartía el mismo precio (10c).

Sin lugar a dudas, las coincidencias y diferencias con este grupo son muchas, en particular en lo que se refiere a las Bellas Artes; no sólo se trata de que la permanencia de Falcini en Montevideo y que sus relaciones personales con los martinfierristas permitan que las notas sobre el arte uruguayo aparezcan alternativamente en uno y otro periódico. Martín Fierro y la Campana comparten las extensas notas dedicadas al Salón Nacional y los artistas seleccionados son muchas veces coincidentes: Raquel Forner, Juan B. Tapia, Alfredo Travascio, Horacio Butler, Héctor Basaldúa. En ambos casos se trata de la valorización de aquellas obras que rompen decididamente con el canon del arte nacional que se afirma sobre la representación de un paisaje "propio"el del interior del país con su descripción de tipos y costumbres. Y en este punto, la reproducción de los dibujos de Ramón Gómez Cornet, con su equilibrio entre elementos formales que valorizan algunas de las conquistas post-cézannianas y su detenimiento en los “seres humildes”, fuera de todo anecdotismo, subrayan una vez más la postura estética de la Campana que es abiertamente rechazada por algunos sectores. ${ }^{17}$

Este fue también el momento del acercamiento entre Atalaya y Emilio Pettoruti -uno de los principales protagonistas de la vanguardia argentina- que coincidió con el alejamiento del pintor platense de Martín Fierro, dato este poco conocido. La Campana anunció y luego dio a conocer una carta de Pettoruti donde hacía público el distanciamiento mencionado. Pero si esto resulta claro, la aparición de otros nombres -Leonardo Estarico y Carlos Astrada- también se deben a su mediación. ${ }^{18}$

\footnotetext{
${ }^{17}$ En carta a Falcini, el 3 de marzo de 1927, At. le informaba: "Un dato. Nos vino de vuelta una Campana, con los dibujos de Cornet corregidos, y con los siguientes comentarios: Manos y pies atrofiados,-cabeza enormes. En los dibujos de la primera página. En la tercera, el croquis de arriba: brazos finos y manos desformes. En el de abajo: Manos y pies desformes, pata "burro". En la cuarta: Brazos, manos y piernas desformes. Al pie de la página: Al señor Yambi tan severo en sus juicios,-hasta para Miguel Ángel en sus dibujos hay que felicitarlo por la publicación de estas incomparables e incomprensibles dibujos: hay que desformar las maravillas de la naturaleza para hacer arte-" (MNBA/42 ) [el énfasis en el original].

${ }^{18}$ Leonardo Estarico, amigo de Pettoruti, era crítico de arte y en 1927 había fundado el Boliche de Arte que organizó durante ese año y el siguiente varias exposiciones. Con Carlos Astrada, el pintor platense estuvo muy ligado en ese momento: el joven filósofo era el director de Clarín. De síntesis
} 
Aunque las filtraciones de Martín Fierro en la Campana o viceversa no impidieron la críticas al grupo vanguardista ("Notas purgativas” 6), los mayores intercambios se produjeron con Boedo: no sólo Yunque y su hermano Juan Guijarro, Tiempo y Riccio, sino también los nombres de Roberto Mariani, Aristóbulo Echegaray, Lisardo Zía, José Salas Subirat, Ernesto Morales, Soto, Pedro J. Vignale fueron los escritores que aparecieron de una u otra forma en sus páginas y le otorgaron a la revista la variedad y dinamismo buscados. Si, por un lado, el adelanto de la Exposición de la actual poesía argentina (1927) recopilada por Vignale y Tiempo (4), marcó el interés por difundir lo que se consideró una de las antologías más importantes dedicadas a los poetas jóvenes, por otro, las encuestas -“¿Cuál es, a su juicio, el peor libro del año?”, con las respuestas fraguadas por Zía y Cascella, o esa breve antología que parodiaba la producción literaria contemporánea -proveniente de un supuesto libro Si non e vero- y los concursos literarios que daban de premio $\$ 1$, fueron conformando el rico espectro de esa visión de la literatura contemporánea desacralizada en la que el castigat ridendo mores cobraba cuerpo de una manera desenfadada. ${ }^{19}$

En este punto, sin embargo, conviene tener presente que los intercambios que venimos señalando tuvieron lugar en el momento en que al suceder a Los Pensadores, Claridad -capitaneada ahora por el aguerrido Leonidas Barletta- cambió decididamente su orientación considerada hasta el momento demasiado "literaria” y adoptó un discurso más dogmático y combativo, reduciendo significativamente el lugar asignado hasta entonces a la poesía. Aunque la línea divisoria entre uno y otro grupo era lábil y puede visualizarse como una delgada membrana fácil de ser traspasada, no obstante se produjo un enfrentamiento que se dirimió en las páginas de ambas revistas. Y esas cuestiones se plantearon no desde las letras, sino desde las artes plásticas y la música: fueron Augusto Gozalvo -antiguo colaborador de Acción de Arte-y Juan Carlos Paz los que generaron con sus notas las más duras réplicas de Barletta. ${ }^{20}$

Pero tal vez lo que resulta más importante para destacar de esta segunda etapa de $L a$ Campana de Palo sea la persistencia en sus páginas de los ideales anarquistas que se verifica en las continuas las referencias a William Ruskin o a Rafael Barret. Al mismo tiempo esa opción política reaparece en varios artículos y notas que se alzan en contra de los totalitarismos, sea de izquierda o de derecha y que se tradujo en la presencia recurrente de los nombres de Henri Barbusse, Romain Rolland y Albert Einstein quienes, en sus llamados a los intelectuales, exigían de ellos una toma de posición. (“A los intelectuales” 1-3). En "El aniversario de la muerte de Lenin” se destacaba, por otra parte, el fracaso

literaria (Córdoba, 1926-27) publicación que marcó la irrupción de la vanguardia en la “docta” Córdoba, acompañada por una serie de intervenciones, entre ellas la visita a la ciudad de la Revista Oral.

${ }^{19}$ Véase también: "Nueva exposición de la actual poesía argentina”,15 (mayo de 1927):5; “1er gran concurso literario de La Campana de Palo”, 12 (febrero 1927): 5; [Armando Cascella y Lizardo Zía]. “Cuál es, a su juicio, el peor libro del año”, 10 (diciembre 1926): 1, “Gansadas greguerizantes”, 13 (marzo 1927): 8.

${ }^{20}$ Véase Gozalvo 4, y Paz 14-15; y en Claridad, los artículos de Barletta: “A un colega”, 15; "Notas Bibliográficas” 16; "Riganelli” 20; y “Una lección” 20. 
evidente en ese trabajar por la violencia y se denunciaba la situación del anarquismo en Rusia y el estado de censura que sufría la prensa opositora (6).

El alertar contra los crímenes del fascismo fue también otra constante, se tratara de la profanación de la tumba de Pietro Gori o de la inmoralidad de quienes reclamaron en nuestro medio el intento de asesinato de Mussollini. Asimismo, el proceso a Sacco y Vanzetti fue seguido con atención, denunciando a los yanquis y exigiendo un boicot a su producción una vez consumada la ejecución de los mártires ("Yanquilandia” 13). La atención prestada a quienes desde América latina asumían una posición política comprometida, llevaron además a reproducir artículos de la revista chilena Claridad y a informar sobre la aparición de Amauta bajo la dirección de José C. Mariátegui y meses después a denunciar su prisión a manos del dictador de turno.

Como vemos, el periódico de Bellas Artes y Polémica se mantuvo fiel a sus principios y a la luz de lo que hemos venido estudiando se puede apreciar mejor la coherencia ya manifiesta en su visualidad y en su contenido. Sin embargo, esto no bastó para asegurarle una vida más prolongada y luego de un número doble de 16 páginas, dejó de aparecer. Un final entrevisto por Giambiagi quien, sin embargo, afirmaba: "Es de sentir que Campana deje de salir, para mí era una cosa prevista o mejor presentida. Pero si es de necesidad una Acción de Arte no es difícil volverla a sacar, en un formato como el Suplemento. Cuando vuelva veremos. Por mi parte siento la necesidad de realizar algo y exponer antes de charlar con la pluma" (228).

El intento no se concretó y cada uno de los integrantes del grupo editor buscó nuevos caminos; en el caso de Atalaya, por primera vez en muchos años, se encontró sin tribuna desde donde expresarse y de hecho, sólo consiguió revertir parcialmente esa situación a partir de su incorporación a Alfar (Montevideo) desde 1929. Por su parte, Ballester Peña se volcó decididamente al catolicismo y, en lo que puede verse como una grave contradicción, siguió colaborando con La Protesta y al mismo tiempo con la recién aparecida Número (Buenos Aires, 1930-1931), del escindido grupo de Criterio.

La revolución del 6 de septiembre de 1930 provocó un nuevo realineamiento de los intelectuales argentinos y fueron otros los espacios a ocupar. Por último, dos años después del golpe de estado, Atalaya -una de las figuras más controvertidas y movilizadoras del campo cultural- falleció.

BiBLIOGRAFÍA

Abad de Santillán, Diego. “La Protesta: su historia, sus diversas fases y su significación en el movimiento anarquista de América del Sur”. Certamen Internacional de La Protesta en ocasión del 30 aniversario de su fundación: 1897 -13 de junio- 1927. Buenos Aires: Editorial La Protesta, 1927.

“Acción de Arte”. [Nota de la Redacción]. Acción de Arte 2/20 (Buenos Aires, noviembrediciembre 1921): 4.

Atalaya. [Alfredo Chiabra Acosta]. Archivo Atalaya (AA). Propiedad Albino Fernández. Buenos Aires. Argentina.

Epistolario Atalaya-Falcini. Donación Falcini. Archivo Documental. Museo Nacional de Bellas Artes (MNBA). Buenos Aires. Argentina. 
“Labor cultural y orientación artística”. La Protesta. Suplemento Semanal 1/23 (Buenos Aires, 19 junio 1922): 2.

“Las Campanas”. La Campana de Palo 1/1 (Buenos Aires, junio 1925): 3-4.

“Exposición José Arato”. La Protesta. Suplemento Semanal (7 julio 1926). Recogido en: Alfredo Chiabra Acosta (Atalaya). 1920-1932. Críticas de Arte Argentino. Buenos Aires: M. Gleizer Editor, 1934. 243-45.

Andreu, Jean. "Lectures anarchistes: la "Librería" de La Protesta, Buenos Aires, juin 1914”. Caravelle. Cahiers du monde hispanique et luso-brésilien 45 (1985): 101106.

Andreu, Jean, Maurice Fraysse y Eva Golluscio de Montoya. Anarkos. Literaturas libertarias de América del Sur. 1900 (Argentina, Chile, Paraguay, Uruguay). Buenos Aires: Corregidor, 1990.

Artundo, Patricia M. Atalaya y el ejercicio de la crítica de arte: 1913-1932. Informe Final de Investigación. Fondo Nacional de las Artes, 2001, inédito.

“El futurismo en Buenos Aires: 1909-1914”. Actas de las Terceras Jornadas de Estudios/Investigaciones. Instituto de Teoría e Historia del Arte “Julio E. Payró”. FFyL. UBA, 1998 (CD-ROM).

Barbusse, Henri, Romain Rolland y Alberto Epstein. “A los intelectuales”. La Campana de Palo. Periódico mensual. Bellas Artes y Polémica 14 (Buenos Aires, abril 1927): 1 y 3.

Barletta, Leónidas. “Renovarse o morir”. Los Pensadores 5/120 (Buenos Aires, abril 1926).

“A un colega bilioso y ultramodernista”. Claridad 6/137 (Buenos Aires, junio 1927).

“Notas bibliográficas”. Claridad 6/138 (Buenos Aires, julio 1927).

“Riganelli”. Claridad 6/142 (Buenos Aires, setiembre 1927).

“Una lección”. Claridad 6/142 (Buenos Aires, setiembre 1927).

Busset, Maurice. La technique moderne du bois gravé. Paris: Librairie Delagrave, 1925.

Corbiére, Emilio J. Los catecismos que leyeron nuestros padres: ideología e imaginario popular en el siglo XX. Buenos Aires: Editorial Sudamericana, 2000.

“Correo del piccolo navío. Serapio Gonzalvo". [El Grupo Editor]. La Campana de Palo. Periódico Mensual de Bellas Artes y Polémica 14 (Buenos Aires, abril 1927): 8.

Díaz, Nilda. “La Campana de Palo. Primera época”. América. Cahiers du C.R.I.C.C.A.L. Le discours culturel dans les revues Latino-Américaines de l'entre deux-guerres. 1919-1939 4-5 (París, 1990): 358-68.

“El aniversario de la muerte de Lenin”. [El Grupo Editor]. La Campana de Palo. Periódico mensual. Bellas Artes y Polémica 11 (Buenos Aires, enero 1927): 6.

Giambiagi, Carlos. Reflexiones de un pintor. Buenos Aires: Editorial Stilcograf, 1972.

Gilimón, Eduado G. Hechos y comentarios. Seguido de "Páginas íntimas” y algunos escritos de varios escritores. Buenos Aires, Montevideo, México: Imprenta B. Puey, [1911].

Gleizes, A. “Estudio sobre el dadaísmo”. Acción de Arte 2/7 (Buenos Aires, agosto 1921): 4. 
Giordano, Carlos. "Boedo y el tema social”. Capítulo: la historia de la literatura argentina. Buenos Aires: Centro Editor de América Latina, 1980.

Golluscio de Montoya, Eva. "Círculos anarquistas y circuitos contraculturales en la Argentina del 1900”. Caravelle. Cahiers du monde hispanique et luso-brésilien 46 (1986): 49-64.

Gozalvo, Augusto. "Proyecto de monumento a Florencio Sánchez”. La Campana de Palo 16 (Buenos Aires, junio 1927): 4.

“La Campana de Palo” [Nota de la Redacción]. La Campana de Palo 1 (Buenos Aires, junio 1925).

Liptchiz. "Baile ruso”. Acción de Arte 2/20 (Buenos Aires, diciembre 1921): 4.

Litvak, Lily. La mirada roja. Estética y arte del anarquismo español (1890-1913). Barcelona: Ediciones del Serbal, 1988.

Maroziuk, Lidia Isabel. "Gráfica crítica e ideario ácrata en una producción urbana: el “Suplemento Semanal de La Protesta” (1922-1930)”. Ciudad/Campo en las artes en Argentina y Latinoamérica. Actas de las 3ras Jornadas de Teoría e Historia del Arte. Buenos Aires: CAIA, 1991. 205-16.

Michel, Pol. “El expresionismo: la joven poesía”. Acción de Arte 2/15 (Buenos Aires, junio 1921): 2.

Muñoz, Miguel Ángel. “Los Artistas del Pueblo: anarquismo y sindicalismo revolucionario en las artes plásticas”. Causas y azares 5 (Buenos Aires, otoño 1997): 116-30.

"Nota” [Nota de El Grupo Editor]. Gustavo Riccio. Un poeta en la ciudad. Buenos Aires: La Campana de Palo, 1926. 95.

“Notas purgativas. Para 'Martín Fierro'. [El Grupo Editor]. La Campana de Palo. Periódico Mensual de Bellas Artes y Polémica 13 (Buenos Aires, marzo 1927): 6.

“Nuestros objetivos” [Nota de la Redacción]. La Protesta. Suplemento Semanal 1 (9 enero 1922): 1.

Oved, Iaacov. El anarquismo y el movimiento obrero en la Argentina. México: Siglo Veintiuno, 1978.

O.P. [ Pelletieri, Osvaldo]. “El peor libro de 1926”. La Opinión Cultural (Buenos Aires, 13-06-1976): 2.

Paz, Juan Carlos. “Algo más acerca de 'La consagración de un músico mediocre: Honneger'”. La Campana de Palo 17 (Buenos Aires, septiembre-octubre 1927): 1415.

Pereyra, Washington. La prensa literaria argentina. 1890-1974. Los años rebeldes. 1920-1929. Buenos Aires: Librería Colonial, 1995.

Quesada, Fernando. "La Protesta una longeva voz libertaria”. Todo es Historia 82 (Buenos Aires, 1974): 74- 96 y 83 (1974): 68-93.

Suriano, Juan. “Ideas y prácticas ‘políticas’ del anarquismo argentino”. Entrepasados. Revista de historia 5/8 (Buenos Aires, 1995): 21-48.

Anarquistas: cultura y política libertaria en Buenos Aires. 1890-1910. Buenos Aires: Manantial, 2001.

Tiempo, César. “César Tiempo y la bohemia literaria de 1926. Los diez centavos fuertes”. La Opinión Cultural (Buenos Aires, 13-06-1976): 2-3. 
Schwartz, Jorge. Las vanguardias literarias: textos programáticos y críticos. México: Fondo de Cultura Económica, 2002. Edición corregida y aumentada (1 $1^{\text {a }}$ edición: 1991).

“Unas cuantas palabras”. [Nota de la Redacción]. La Campana de Palo. Periódico Mensual. Bellas Artes y Polémica 7 (Buenos Aires, septiembre 1926): 1.

“Una aventura editorial”. [El Grupo Editor]. La Campana de Palo. Periódico Mensual. Bellas Artes y Polémica 10 (Buenos Aires, diciembre 1926): 3.

Vignale, Pedro J. y C. Tiempo. “Exposición de la actual poesía argentina”. La Campana de Palo. Periódico mensual. Bellas Artes y Polémica 10 (Buenos Aires, diciembre 1926): 4.

Viguera, Aníbal. "Participación electoral y prácticas políticas de los sectores populares en Buenos Aires, 1912-1922”. Entrepasados. Revista de historia 1 (Buenos Aires, 1991): 5-33.

Walzer, Michael. La compañía de los críticos. Intelectuales y compromiso político en el siglo XX. Buenos Aires: Nueva Visión, 1993.

"Yanquilandia”. [El Grupo Editor]. La Campana de Palo. Periódico mensual. Bellas Artes y Polémica 17 (Buenos Aires, septiembre-octubre 1927): 13.

Yunque, Álvaro. Zancadillas. Buenos Aires: Editorial La Campana de Palo, 1926.

“Carlos Giambiagi”. Cuadernos de Cultura 52 (Buenos Aires, marzo-abril 1961): 107-12. 